Artículo

\title{
Influencia de la fertilización en rendimiento y calidad de grano del centeno en la zona árida de Sonora, México
}

\author{
Juan Pedro López Córdova ${ }^{1}$ \\ Francisco Rodríguez Félix ${ }^{1}$ \\ Francisco Javier Wong Corral ${ }^{1}$ \\ Francisco Ramírez Reyes ${ }^{2}$ \\ Edgar Omar Rueda Puente ${ }^{2}$ \\ Guadalupe Amanda López Ahumada ${ }^{1 \S}$ \\ ${ }^{1}$ Departamento de Investigación y Posgrado en Alimentos-Universidad de Sonora. Hermosillo, Sonora, \\ México. (juancordovape@gmail.com; frodriguez@guayacan.uson.mx; francisco.wong@unison.mx. \\ ${ }^{2}$ Departamento de Agricultura y Ganadería-Universidad de Sonora. Carretera a Bahía de Kino km 20.5, \\ Hermosillo, Sonora, México. (framirez@guaymas.uson.mx; erueda04@ santana.uson.mx). \\ ${ }^{\S}$ Autor para correspondencia: amanda.lopez@unison.mx.
}

\section{Resumen}

En la zona árida del noroeste de México, el cultivo del centeno no se encuentra establecido y es necesario generar su conocimiento en materia agronómica. El objetivo del presente estudio consistió en determinar la factibilidad del cultivo de centeno considerando dosis de fertilización nitrogenada evaluando el rendimiento y sus componentes de calidad de grano. El trabajo de investigación se desarrolló en el municipio de Hermosillo, Sonora. La fecha de siembra establecida fue el 15 diciembre del año 2016 utilizando un sistema de riego por goteo. La variedad de centeno evaluada se denomina como Criollo Tlaxcala. Los tratamientos de fertilización consistieron en: 1) testigo sin agregado de $\mathrm{N}(\mathrm{N} 0), 2) 80 \mathrm{~kg} \mathrm{~N} \mathrm{ha}^{-1}$ y el 3) $150 \mathrm{~kg} \mathrm{~N}^{-1}$. Los resultados indican que conforme aumenta la dosis de fertilización, ésta influye significativamente, en el rendimiento y calidad de grano. Asimismo, los resultados arrojan que el contenido de proteína supera en $11 \%$ a aquellos reportados en diferentes países en centeno.

Palabras clave: cereales secundarios, grano, proteína.

Recibido: junio de 2019

Aceptado: septiembre de 2019 


\section{Introducción}

En los países en desarrollo, la demanda de cereales ha crecido con mucha mayor rapidez que la producción y es probable que aumente esta dependencia de las importaciones (FAO, 2015). En el año 2030, los países en desarrollo podrían importar anualmente 265 millones de toneladas de cereales; es decir, 14\% de su utilización. El consumo de cereales secundarios ha crecido rápidamente, impulsado sobre todo por el uso creciente como alimento en los países en desarrollo. En un futuro, puede aumentar su consumo con mayor rapidez que los cultivos tradicionales como arroz, trigo y maíz, dependiendo del crecimiento del sector alimentario y pecuario (FAO, 2015).

México resalta en los pronósticos globales en importaciones de cereales secundarios principalmente de centeno; Sus importaciones aumentaran en 15\%, lo que significa 23.8 millones de toneladas, entre 2015 y 2025 (USDA, 2015). Las zonas áridas son de las áreas más importantes de producción agrícola a nivel mundial. Las características más importantes de estas áreas son la indisponibilidad del recurso agua, altas y bajas temperaturas e intrusión salina, entre otras (Mazuela Aguilar, 2013; Andrade et al., 2017). El 60\% de los alimentos a nivel mundial se producen en zonas áridas.

En la República Mexicana, una de las zonas de relevancia en la producción de alimentos, es el noroeste de México, conformado por los estados de Baja California, Baja California Sur, Sonora, Sinaloa y Chihuahua (Martínez et al., 2016; Andrade et al., 2017). Entre los principales cultivos con interés comercial de exportación son los siguientes: trigo, ajonjolí, cártamo, garbanzo, sorgo, maíz, algodón, vid, nogal, sandía, tomate, entre otros productos. En Sonora la superficie destinada para estos cultivos es de 614606 ha.

La fertilización nitrogenada es uno de los factores más importantes en el crecimiento y desarrollo de los cultivos de cereales. La disponibilidad de $\mathrm{N}$ para la planta es indispensable por ser un componente básico de las moléculas orgánicas involucradas en el crecimiento y desarrollo vegetal (Salas, 2003). La búsqueda de cereales secundarios alternativos con propiedades nutricionales benéficas para el consumo humano como el centeno, es de suma importancia sobre todo cuando se trata de producirlos en ambientes áridos en los cuales existen factores adversos como condiciones edáficas y climáticas que merman la productividad de los cultivos (Murillo et al., 2001; Liukkonen et al., 2003; Bushuk, 2004).

El contenido de proteína del grano de centeno se encuentra en el rango de 6.5 a $14.5 \%$ y es dependiente de las condiciones de manejo agronómico del cultivo, aunque el contenido de proteína del grano ha sido reportado que es influenciado ante todo por el genotipo (Hansen et al., 2004; Arendt y Zannini, 2013). Desde el punto de vista nutricional las proteínas de centeno están reconocidas ser superiores a las de trigo y otros granos de cereal debido al mejor balance de aminoácidos esenciales (Arendt y Zannini, 2013). En la zona árida del noroeste de México, está el interés de establecer el cultivo del centeno.

Sin embargo, es necesario ampliar el conocimiento en materia agronómica. El objetivo del presente estudio consistió en determinar la factibilidad del cultivo de centeno considerando diferentes dosis de fertilización nitrogenada con relación al rendimiento y sus componentes de calidad de grano. 


\section{Materiales y métodos}

\section{Descripción del sitio experimental o área de estudio}

El trabajo de investigación se desarrolló en el campo experimental del Departamento de Agricultura y Ganadería (DAG) de la Universidad de Sonora que se localiza con las coordenadas $29^{\circ} 00^{\prime} 46.2^{\prime \prime}$ latitud norte, $111^{\circ} 08^{\prime} 03.1$ ' latitud oeste a $146 \mathrm{msnm}$. Predomina un clima subtropico árido semicálido, con precipitación y temperatura media anual de $320.8 \mathrm{~mm}$ y $23.1^{\circ} \mathrm{C}$, respectivamente (INIFAP, 2010). El área experimental se estableció en un suelo con textura franco arenosa, caracterizada por un contenido de $49.08 \%$ de arena, $32.12 \%$ de limo y $18.8 \%$ de arcillas. La fecha de siembra establecida fue en el ciclo otoño-invierno 15 diciembre de 2016-2017. Se utilizó un sistema de riego por goteo.

La preparación del suelo se realizó mecánicamente con un barbecho y un posterior pase de rastra. La variedad de centeno evaluada fue 'Criollo Tlaxcala' donada por la Asociación Agrícola de Tlaxcala, México. El método de siembra en forma manual a chorrillo en doble hilera, con una dosis de $120 \mathrm{~kg} \mathrm{ha}^{-1}$. Al momento de la siembra se aplicó $80 \mathrm{~kg} \mathrm{ha}^{-1}$ de fósforo (P) utilizando el fosfato mono amónico (12N-61P-0K). Los tratamientos de fertilización consistieron en: 1) testigo sin $\mathrm{N}$ (N 0), 2) $80 \mathrm{~kg} \mathrm{~N} \mathrm{ha}^{-1}$ y 3) $150 \mathrm{~kg} \mathrm{~N} \mathrm{ha}^{-1}$, los fertilizantes fueron aplicados fraccionadamente en la etapa fisiológica de embuche (70\%) y en floración-antesis (30\%), la fuente utilizada fue la de nitrato de amonio (33N-03P-00K).

Se realizó un control de maleza manual del cultivo para evitar competencia por el $\mathrm{N}$ utilizado. No se registró presencia de plagas y enfermedades durante todo el periodo del ciclo vegetativo.

\section{Diseño y análisis estadístico}

El experimento se estableció con un diseño en bloques al azar; se implementaron 3 fertilizaciones nitrogenadas ( 080 y $150 \mathrm{~kg} \mathrm{ha}^{-1}$ ), se realizaron 4 repeticiones en la parcela para su posterior análisis. La parcela experimental constó de un área de $30 \mathrm{~m}^{2}$. Los datos obtenidos fueron analizados mediante un análisis de varianza (Anova). La prueba de Tukey, se utilizó para analizar las diferencias entre las medias de tratamiento específicos. Se utilizó el software estadístico InfoStat (Di Rienzo et al., 2015) para Windows.

\section{Etapas fenológicas, variables de rendimiento y sus componentes}

Las etapas fisiológicas evaluadas en el presente estudio se registraron en días después de siembra (DDS), consistieron en emergencia, amacollamiento, embuche, espigamiento, antesis y madurez fisiológica. La descripción de las etapas se detalla en el (Cuadro 1) de acuerdo a Solís Moya et al. (2004) utilizando la escala de Zadoks et al. (1974).

Al finalizar el experimento, se midió el número de espigas por $\mathrm{m}^{2}$ y se realizó la cosecha de biomasa y grano. Las variables evaluadas fuero biomasa total, peso hectolitrico, peso de mil granos y proteína de grano. El rendimiento de grano (RG) se determinó con el peso de la semilla cosechada en gramos, dividiéndolo entre la superficie cosechada y expresado en $\mathrm{t} \mathrm{ha}^{-1}$. 
Cuadro1. Descripción de las etapas fenológicas evaluadas.

\begin{tabular}{ll}
\hline Variable & Medición \\
\hline Amacollo & Aparición del primer amacollo 50\% de las plántulas \\
Embuche & Cuando 50\% de la vaina de las plantas están engrosadas o hinchadas \\
Espigamiento & Cuando 50\% de las espigas estuvieran expuestas \\
Antesis & Cuando se expusieron las anteras de la parte media de la espiga en 50\% \\
Madurez fisiológica & $\begin{array}{l}\text { Cuando 50\% de las plantas perdió el color verde característico } 80 \% \text { de } \\
\text { sus hojas, tallos y espiga. }\end{array}$ \\
\hline
\end{tabular}

La biomasa total (BIO) se obtuvo en la etapa vegetativa de madurez fisiológica de manera manual para ello utilizando una rozadera, el corte de la planta se hizo aproximadamente a una altura de 5 $\mathrm{cm}$ sobre la superficie del suelo, calculando el rendimiento en toneladas por hectárea ( $\left.\mathrm{t} \mathrm{ha}^{-1}\right)$. Para la determinación de peso hectolitrico (PHL) se utilizó un homogeneizador (modelo Boerner 34, Seedburo) después se pesó la muestra en una balanza analítica (modelo 8850, Seedburo) determinando el valor en $\left(\mathrm{kg} \mathrm{hl}^{-1}\right)$ siguiendo la metodología de la (AACC, 2000).

Para la variable peso de mil granos (PMG) se consideraron 4 réplicas, las cuales fueron pesadas en una balanza analítica (OHAUS, modelo AR N 2140), registrándose en gramos (gr). Por otra parte, la proteína (PRO) de grano se identificó de acuerdo al método 46-13.01 de la (AACC, 2000) utilizando un determinador de nitrógeno (LECO FP-528. Leco Corporation) aplicando un factor de conversión de (N x 6.25) (Wrigley et al., 2010).

Se calcularon los grados días de crecimiento (GDC) acontecidos desde la siembra hasta madurez comercial. Para estimar la duración de las distintas etapas fenológicas se utilizó la propuesta de Ma y Smith (1992), considerando la siguiente ecuación.

$\mathrm{GD}=\sum_{\mathrm{i}=1}^{\mathrm{n}}\left(\mathrm{T}_{\mathrm{ai}}-\mathrm{T}_{\mathrm{b}}\right)$

Donde: $\mathrm{T}_{\mathrm{ai}}$ representa el promedio de las temperaturas máximas y mínimas diarias del aire; $\mathrm{T}_{\mathrm{b}}$ a la temperatura base en donde la planta detiene su crecimiento, que por lo general la mayoría de los cereales consideran un valor de $0{ }^{\circ} \mathrm{C}$ (Cao y Moss, 1989; Kernich y Halloran, 1996).

Los datos de precipitación pluvial, temperaturas máximas y mínimas del aire durante el desarrollo del experimento, se tomaron de una estación automática marca VAISALA modelo (WXT510), ubicada a $20 \mathrm{~m}$ de distancia del lugar en donde se encuentra la siembra experimental.

\section{Resultados y discusión}

\section{Fenología y condiciones climáticas.}

Con relación a los grados días calor (GDC) las representaciones de las fases fenológicas de manera general hasta madurez comercial se encuentran representados en la (Figura 1), los análisis de varianza no muestran una diferencia significativa. Esto refleja poca variación en la fenología del 
centeno en los tres tratamientos de fertilización nitrogenada. La demanda total del período térmico del cultivo a madurez fisiológica fue de 2384 GDC en 128 días después de siembra. En la (Figura 2), se observa que las primeras etapas del cultivo se desarrollaron en un ambiente entre los 7 y 30 ${ }^{\circ} \mathrm{C}$ con precipitaciones no mayores a los $10 \mathrm{~mm}$ como media mensual.

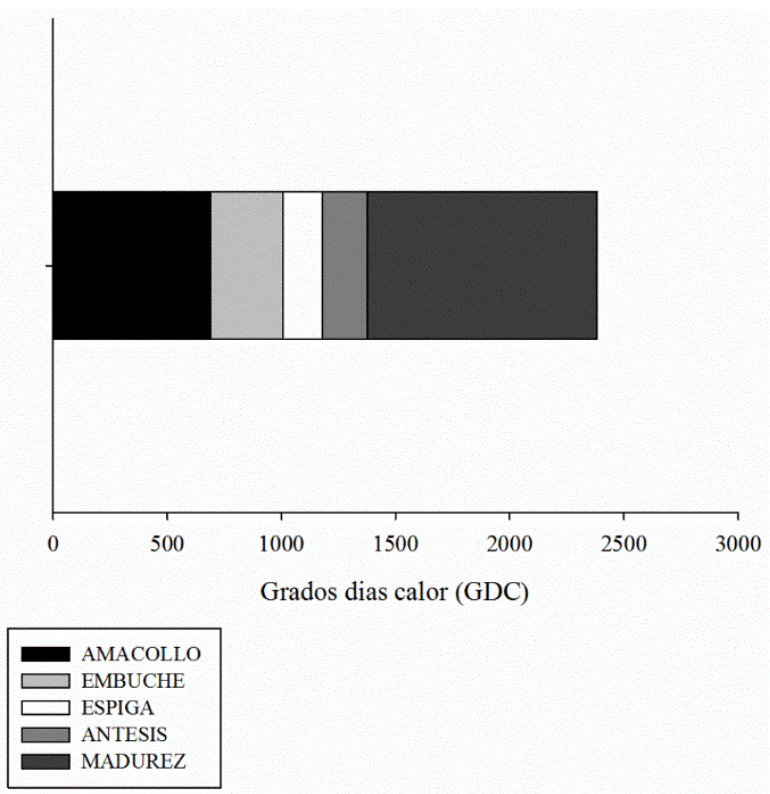

Figura 1. Duración de las principales etapas en función de grados días calor (GDC), para el ciclo agrícola invierno-primavera 2016-2017.

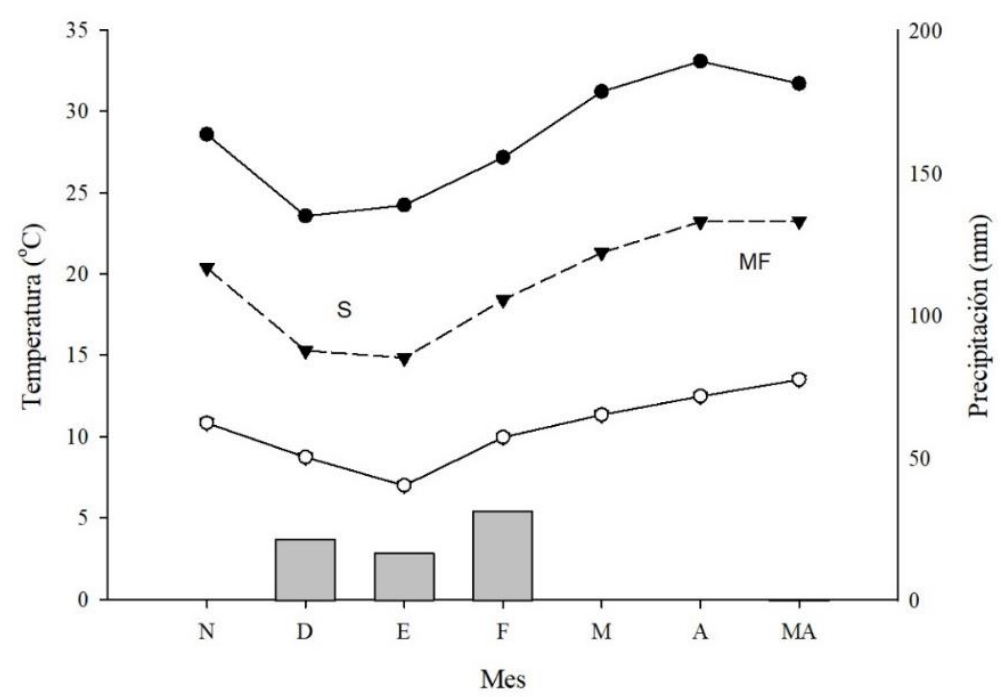

Figura 2. Precipitación $(\square)$ y temperaturas $\left({ }^{\circ} \mathrm{C}\right)$ máximas $(\bullet)$, mínimas $(\circ)$, promedio mensual $(\nabla)$. $\mathrm{S}=$ siembra, 15 de diciembre 2016; MF= madures fisiológica, 20 de abril 2017.

El comportamiento de este cultivar en GDC es similar a los de Ballesteros et al. (2015) en triticale, donde resultaron 2137 GDC y 2307 GDC en dos ciclos experimentales (Ballesteros et al., 2015). Por otra parte, López et al. (2018) reportaron un trabajo con centeno en la temporada invierno- 
primavera en la localidad de Hermosillo, Son. Con resultados no similares a los obtenidos en este estudio, obteniendo 2274 GDC y 112 DDS hasta madurez fisiológica. El mayor número de GDC y DDS en el presente trabajo se atribuye al establecimiento del cultivo en fechas donde las temperaturas del aire e incidencia de radiación solar son predominantemente más bajas.

\section{Rendimiento de granos y componentes}

Los tratamientos (T) con dosis de 0,80 y $150 \mathrm{~kg} \mathrm{ha}^{-1}$, desarrollaron diferencias estadísticas en todas las variables excepto $\mathrm{PH}$ observando diferencias en el RG, PRO, NGE, BIO y al $p \leq 0.05$ en el $(\mathrm{NEPM})$ y $(\mathrm{PMG})$, la fertilización $(\mathrm{N})$ es un factor importante para incrementar el rendimiento y sus componentes (Cuadro 2).

Cuadro 2. Valores de $\mathrm{F}$ en el análisis de varianza combinado para el rendimiento de centeno en tres tratamientos de fertilización.

\begin{tabular}{ccccccc}
\hline Fuente de variación & BIO & NEPM & PMG & PHL & PRO & RG \\
\hline Repetición & 0.6 & 0.26 & 1.34 & 0.28 & 1.44 & 0.13 \\
Fertilización & $17.9^{*}$ & $4.59^{*}$ & $10.3^{*}$ & $0.58 \mathrm{~ns}$ & $17.15^{* *}$ & $27.06^{* *}$ \\
Error (CM) & 456394 & 343.47 & 0.95 & 0.19 & 0.06 & 17256.07 \\
CV $(\%)$ & 6.12 & 9.7 & 2 & 0.6 & 1.51 & 6.33 \\
\hline
\end{tabular}

$\mathrm{BIO}=$ biomasa; $\mathrm{NGE}=$ número de granos por espiga; $\mathrm{NEPM}=$ número de espigas por metro cuadrado; $\mathrm{PMG}=$ peso de mil granos; $\mathrm{PHL}=$ peso hectolitrico; $\mathrm{PRO}=$ proteína total; $\mathrm{RG}=$ rendimiento de grano; ${ }^{*}=p \leq 0.05 ;{ }^{* *}=p \leq 0.01 ; \mathrm{ns}=\mathrm{no}$ significativa; $\mathrm{CV}=$ coeficiente de variación.

\section{Rendimiento}

La comparación de medias entre tratamientos para la variable de rendimiento de grano $\left(\mathrm{kg} \mathrm{ha}^{-1}\right)$ mediante la prueba de Tukey $(p \leq 0.05)$ en la (Figura 3), señalan que se obtuvo una diferencia significativa $p \leq 0.01$ entre los tres niveles de fertilización. Por lo tanto, el rendimiento de grano aumentó a medida que se incrementó la cantidad de nitrógeno desde el control hasta $150 \mathrm{~kg} \mathrm{ha}^{-1}$. El tratamiento con $150 \mathrm{~kg} \mathrm{ha}^{-1} \mathrm{~N}$ obtuvo un promedio de $2385 \mathrm{~kg} \mathrm{ha}^{-1}$ presentando el máximo rendimiento, en contraste con el testigo absoluto promedio de $1965 \mathrm{~kg} \mathrm{ha}^{-1}$, dosis bajas de nitrógeno pueden afectar o inducir el estado fenológico en el cultivo, afectando a su vez, el inicio de la antesis y la floración (Fois et al., 2009).

Los resultados obtenidos en el presente estudio concuerdan con Bakhsh et al. (1999); Rustam y Yasin (1991), donde informaron que un aumento en las dosificaciones de nitrógeno tiene repercusiones positivas en comportamiento del rendimiento de grano. Por otra parte, los rendimientos obtenidos en todos los tratamientos evaluados fueron superiores a la media reportados por López et al. (2018), donde este autor trabajo con centeno obteniendo una media general de $1965 \mathrm{~kg} \mathrm{ha}^{-1}$. Este efecto nos indica que a medida que la fecha de siembra sea más cálida, las condiciones ambientales son menos favorables para la producción de este grano. 


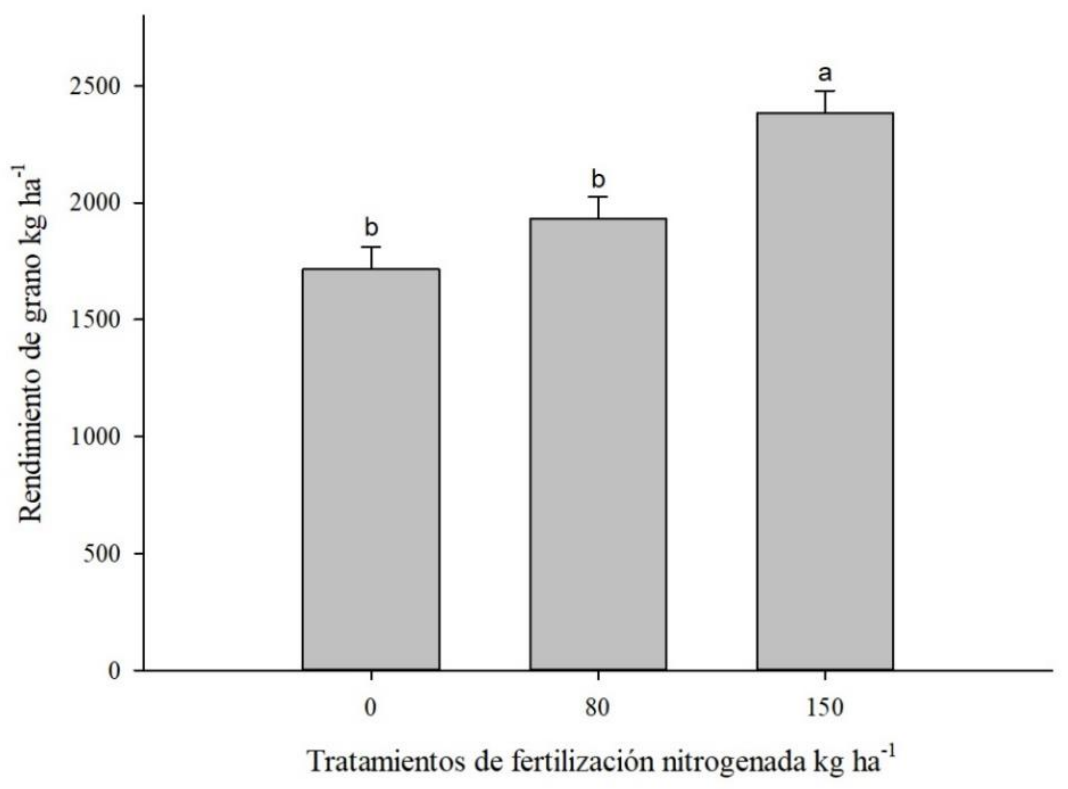

Figura 3. Efecto de la dosificación del nitrógeno sobre los componentes de rendimiento de grano.

El número de espigas por metro cuadrado y el peso de mil granos mantuvieron un efecto positivo lineal a medida que se incrementaba las dosificaciones del fertilizante nitrogenado. Langer y Liew (1973), evaluando los efectos de la variación en la suplementación del nitrógeno aplicado a diferentes etapas del cultivo en trigo, revelaron que el número de espiguillas se incrementa con el incremento de nitrógeno aplicado al cultivo, produciendo a su vez un número mayor de hojas y por lo tanto, afectando al rendimiento. Además, Ali et al. (2003) evaluando dosis de nitrógeno sobre el rendimiento de grano, encontraron que el rendimiento de grano depende en su mayoría del peso de grano y también al número de espigas por metro cuadrado (Figura 4).

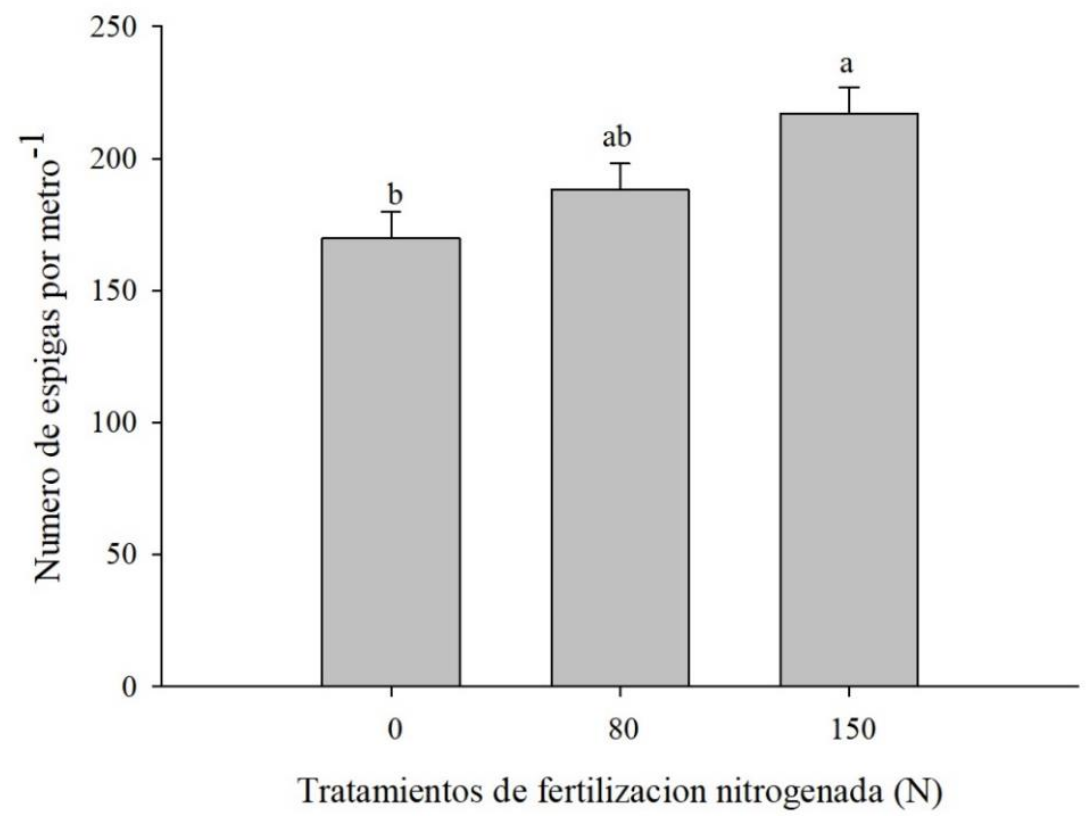

Figura 4. Efecto de la dosificación del nitrógeno sobre el número de espigas por metro. 
Los resultados revelan que para los tratamientos con una dosificación de $150 \mathrm{~kg} \mathrm{ha}^{-1}(\mathrm{~N})$ (Figura 5) la producción de biomasa a madurez fisiológica fue la más alta $(p \leq 0.05)$ con $12513 \mathrm{~kg} \mathrm{ha}^{-1}$.

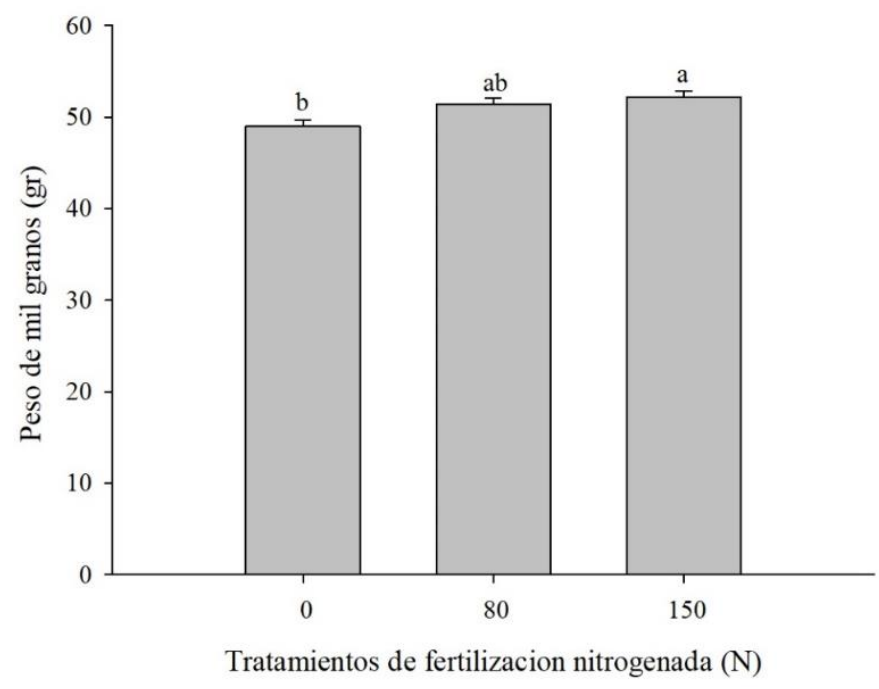

Figura 5. Efecto de la dosificación del nitrógeno sobre el peso de mil granos.

El incremento en la producción de biomasa en los cultivares de centeno y trigo se ha observado durante diversas investigaciones que corresponde con un aumento en los niveles del nutriente nitrógeno inorgánico presentes en las propiedades edafológicas (Balkcom et al., 2011) (Figura 6).

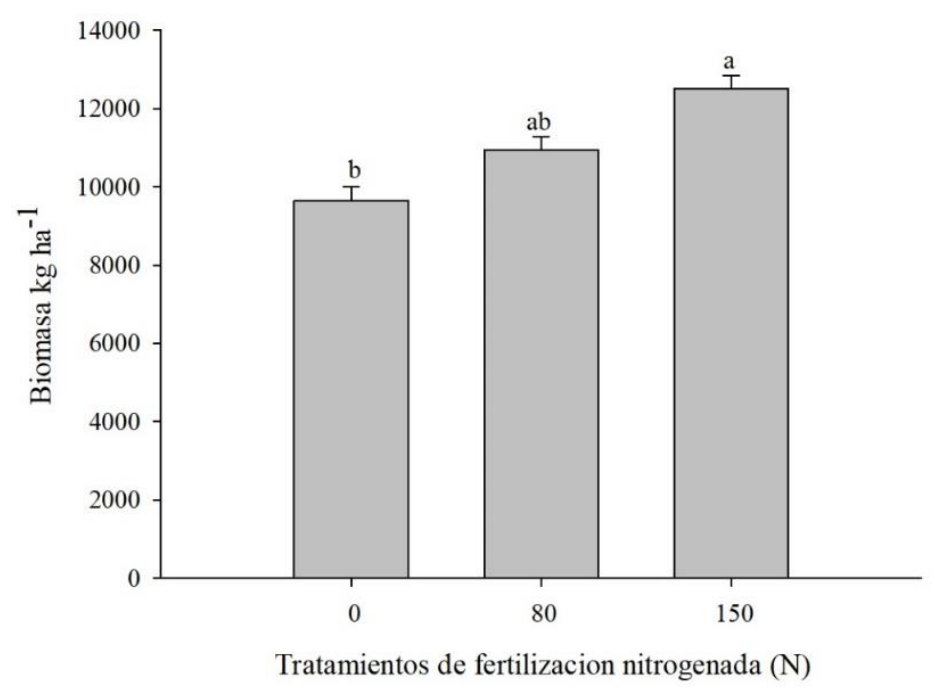

Figura 6. Efecto de la dosificación del nitrógeno sobre el componente de biomasa ( $\left.\mathrm{kg} \mathrm{ha}^{-1}\right)$.

En el Cuadro 2 se describe la relación del peso hectolítrico con la fertilización nitrogenada este factor no tuvo una significancia $p \leq 0.05$; por lo tanto, no se observó tampoco un efecto en los tres niveles de fertilización, lo que nos indica la estabilidad fenotípica para estas características. Estos resultados, reafirman la explicación de Mellado et al. (2008), donde indica que la aplicación de nitrógeno con tan solo $50 \mathrm{~kg} \mathrm{ha}^{-1}$ mejora el peso hectolítrico de centeno en $1 \mathrm{~kg} \mathrm{hL}^{-1} \mathrm{y}$ dosis mayores a este nutriente no hicieron variar significativamente esta característica. 
La cantidad de nitrógeno también afecto significativamente la calidad de proteína del grano. La interacción entre las tres dosis de nitrógeno y el contenido proteínico fue significativa $p \leq 0.05$. Evidentemente, el contenido de proteína se incrementó con las dosificaciones del fertilizante. Se observó un contenido máximo (16.5\%) de proteína con la dosis de $150 \mathrm{~kg} \mathrm{ha}^{-1}$, el contenido mínimo de proteína de grano (14.78\%) se presentó con la dosis de $0 \mathrm{~kg} \mathrm{ha}^{-1}$. Esto puede explicarse por la mayor disponibilidad de nitrógeno para la síntesis de aminoácidos (Figura 7) y a su vez en la posterior síntesis de proteínas.

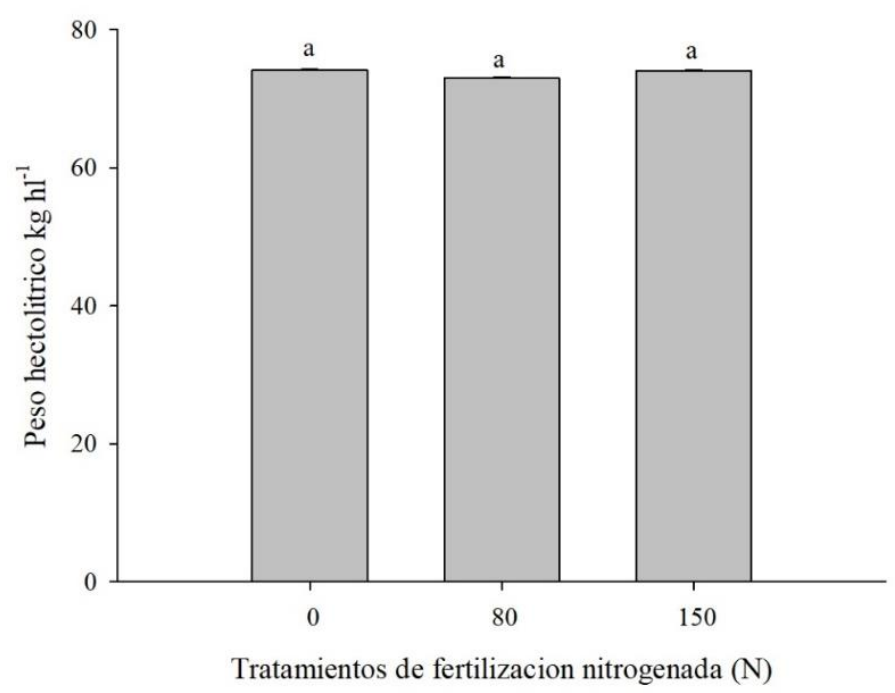

Figura 7. Efecto de la dosificación del nitrógeno sobre el peso hectolitrico $\left(\mathrm{kg} \mathrm{hL}^{-1}\right)$.

Se ha observado un incremento lineal en el porcentaje de proteína a medida que se extendía el rango de nitrógeno en trigo panadero (Figura 8).

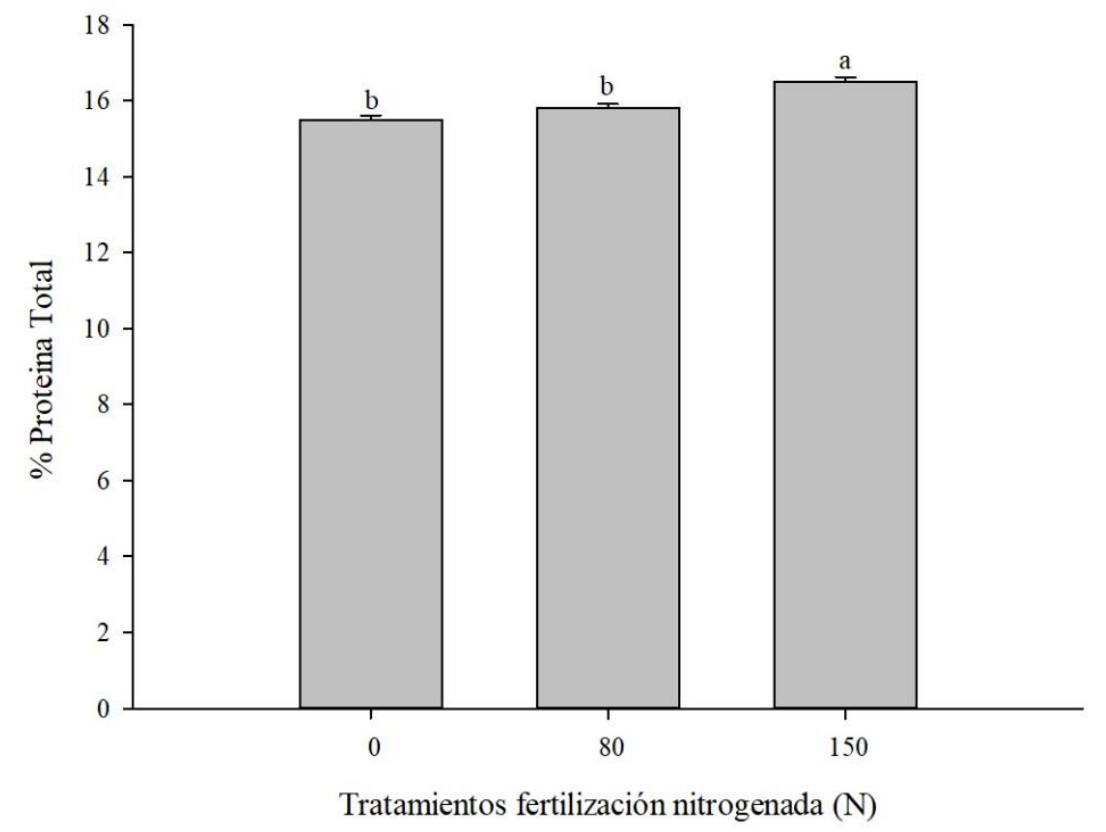

Figura 8. Efecto de la dosificación del nitrógeno sobre el componente de proteína. 


\section{Conclusiones}

Con la realización del presente estudio, fue posible ampliar el conocimiento del comportamiento agronómico y calidad en el cultivo de centeno, bajo la influencia de tres dosis de fertilización nitrogenada, bajo condiciones de campo de zonas áridas en el noroeste de México. Los resultados arrojaron que la variedad de centeno evaluada como Criollo Tlaxcala en la fecha de siembra del 15 diciembre del 2016-2017, con un sistema de riego por goteo, al incrementar la dosis de fertilización de 0 a $150 \mathrm{~kg} \mathrm{ha}^{-1}$, presentan valores significativos positivos en la variable de rendimiento, calidad de grano y biomasa.

De igual forma, se concluye que el contenido de proteína obtenido bajo las condiciones propuestas, supera a aquellos reportados en diferentes países donde se dedican a la producción de centeno. Se recomienda que en posteriores estudios deban ser tomados en cuenta, factores relacionados con fechas de siembra, condiciones limitantes del recurso agua y salinidad.

\section{Agradecimientos}

Agradecemos a la Dra. María Isabel Silveira Gramont por su apoyo en el diseño de experimentos de este estudio y del análisis estadístico. Así como, a la Dra. Concepción Lorenia Medina Rodríguez por su apoyo en los análisis de proteína.

\section{Literatura citada}

AACC International. 2000. Approved Methods of the AACC. Methods 44-20, 46-13, 55-10.01. The association, St Paul, MN.

Ali, L.; Mohy, U. D. Q. and Ali, M. 2003. Effect of different doses of nitrogen fertilizer on the yield of wheat. Inter. J. Agric. Biol. 5(4):438-439.

Andrade, B. G.; Manelik, G. L. A.; Cervantes, D. L.; Aíl, C. C. E.; Borboa, F. J. y Rueda, P. E. O. 2017. Estudio del potencial biocontrolador de las plantas autóctonas de la zona árida del noroeste de México: control de fitopatógenos. Revista de la Facultad de Ciencias Agrarias. Universidad Nacional de Cuyo. 49(1):127-142.

Arendt, E. K. and Zannini, E. 2013. Cereal grains for the food and beverage industries. Cambridge. Woodhead Publishing Series in Food Science. Technol. Nutr. 485 p.

Bakhsh, A.; Gurmani, A. H.; Bhatti, A. U. and Rehman, H. 1999. Effect of balanced application of N, P and K on the grain yield of wheat. Sarhad. J. Agri. 15(1):453-457.

Balkcom, K. S.; Massey, J. M.; Mosjidis, J. A.; Price, A. J. and Enloe, S. F. 2011. Planting date and seeding rate effects on sunn hemp biomass and nitrogen production for a winter cover crop. Inter. J. Agron. 2011.

Ballesteros, R. E.; Morales, R. E. J.; Franco, M. O.; Santoyo, C. E.; Estrada, C. G. y Gutiérrez, R. F. 2015. Manejo de fertilización nitrogenada sobre los componentes del rendimiento de triticale. Rev. Mex. Cienc. Agríc. 6(4):721-733.

Bushuk, W. 2004. 'Rye'. In: Wrigley, Walker, C. C. and Corke, H. (Eds.) Encyclopedia of grain science, Vol.3, Oxford, UK, Elsevier Ltd. 85-91.

Cao, W. and Moss, D. N. 1989. Temperature effect on leaf emergence and phyllochron in wheat and barley. Crop Sci. 29(4):1018-1021. 
Di, R. J. A.; Casanoves F.; Balzarini, M. G.; González, L.; Tablada, M. y Robledo, C. W. InfoStat versión 2015. Grupo InfoStat, FCA. Universidad Nacional de Córdoba, Argentina. http://www.infostat.com.ar.

FAO. 2010. Agricultura mundial: hacia los años 2015-2030. Informe resumido. 160 p.

Fois, S.; Motzo, R. and Giunta, F. 2009. The effect of nitrogenous fertilizer application on leaf traits in durum wheat in relation to grain yield and development. Field Crops Res. 110(1):69-75.

Hansen, H. B.; Møller, B.; Andersen, S. B.; Jørgensen, J. R. and Hansen, A. 2004. Grain characteristics, chemical composition, and functional properties of rye (Secale cereale L.) as influenced by genotype and harvest year. J. Agric. Food Chem. 52(8):2282-2291.

INIFAP. 2010. Instituto Nacional de Investigaciones Forestales, Agrícolas y Pecuarias. Guía técnica para el área de influencia del campo experimental Costa de Hermosillo, Hermosillo, Sonora, México. 8-10 pp.

Kernich, G. C. and Halloran, G. M. 1996. Temperature effects of the duration of the spikelet growth phase and spikelet abortion in barley. J. Agron. Crop Sci. 176(1):23-29.

Langer, R. H. M. and Liew, F. K. Y. 1973. Effects of varying nitrogen supply at different stages of the reproductive phase on spikelet and grain production and on grain nitrogen in wheat. Austr. J. Agric. Res. 24(5):647-656.

Liukkonen, K. H.; Katina, K.; Wilhelmson, A.; Myllyma, K. O.; Lampi, A. M.; Kariluoto, S.; Piironen, V.; Heinonen, S. M.; Nurmi, T.; Adlercreutz, H.; Peltoketo, A.; Pihlava, J. M.; Hietaniemi, V. and Poutanen, K. 2003. Process-induced changes on bioactive compounds in whole grain rye. Proceedings Nutr. Soc. 62(1):117-122.

López, C. J. P.; Rueda, P. E. O.; Vargas, L. J. M.; Wong, C. F. J. and López, G. A. 2018. Rendimiento y calidad de grano en centeno (Secale cereale L.) bajo diferentes condiciones de fertilización en el estado de Sonora, México. Biotecnia. 20(3):76-82.

Ma, B. L. and Smith, D. L. 1992. Apical development of spring barley under field conditions in northeastern North America. Crop Sci. 32(1):144-149.

Martínez, R. F. E.; Cervantes, D. L.; Aíl, C. C. E.; Hernández, M. L. G.; Sánchez, C. L. D. T. y Rueda, P. E. O. 2016. Hongos fitopatógenos asociados al tomate (Solanum Lycopersicum L.) en la Zona Árida del noroeste De México: la importancia de su diagnóstico. Eur. Sci. J. 12(18): 232-256.

Mazuela, Á. P. C. 2013. Agricultura en zonas áridas y semiáridas. Idesia (Arica). 31(2):3-4.

Mellado, Z. M.; Matus, T. I. y Madariaga, B. R. 2008. Antecedentes sobre el centeno, en Chile y otros países. Chillán, Chile. Instituto de Investigaciones Agropecuarias (IIA). Boletín INIA número182. $64 \mathrm{p}$.

Murillo, B.; Escobar, A.; Fraga, H. y Pargas, R. 2001. Rendimiento de grano y forraje de líneas de triticale y centeno en Baja California Sur, México. Rev. Fitotec. Mex. 24(2):145-153.

Rustam, A. and Yasin, M. 1991. Response of wheat to nitrogen and phosphorus fertilization. Pak. J. Agri. Res. 12:30-133.

Salas, C. 2003. Nutrición mineral de plantas y el uso de fertilizantes. In: Meléndez, G. y Molina, E. (Eds.). Fertilizantes: características y manejo. Centro de Investigaciones Agronómicas (CIA). UCR, San José. 1-19 pp.

Solís, M. E.; Hernández, M. M.; Borodanenko, A.; Aguilar, A. J. L. y Grajeda, C. Ó. A. 2004. Duración de la etapa reproductiva y el rendimiento de trigo. Rev. Fitotec. Mex. 27(4):323-332.

USDA. 2015. United States Department of Agricultural. Agricultural projections to 2024. 97 p. 
Wrigley, C. W. and Bushuk, W. 2010. Rye and triticale: characteristics and quality requirements. Cereal grains: assessing and managing quality. 112-140.

Zadoks, J. C.; Chang, T. T. and Konzak, C. F. 1974. A decimal code for the growth stages of cereals. Weed research. 14(6):415-421. 